Société d'histoire de la révolution de 1848 et des

révolutions du XIXe siècle

\title{
Retour sur l'ancien esprit du capitalisme
}

Jean-Pierre Hirsch

URL : http://journals.openedition.org/rh19/312

DOI : $10.4000 /$ rh 19.312

ISSN : $1777-5329$

Éditeur

La Société de 1848

Édition imprimée

Date de publication : 1 décembre 2001

Pagination : 87-104

ISSN : 1265-1354

Référence électronique

Jean-Pierre Hirsch, «Retour sur l'ancien esprit du capitalisme », Revue d'histoire du XIXe siècle [En

ligne], 23 | 2001, mis en ligne le 07 mars 2008, consulté le 01 mai 2019. URL : http://

journals.openedition.org/rh19/312; DOI : 10.4000/rh19.312

Ce document a été généré automatiquement le 1 mai 2019.

Tous droits réservés 


\title{
Retour sur l'ancien esprit du capitalisme
}

\author{
Jean-Pierre Hirsch
}

1 Il fut un temps où l'ancien monde industriel, celui du XIX siècle, apparaissait bien en ordre dans nos têtes. Et il en reste quelque chose : nombre d'analyses, pour comprendre ce qui nous arrive à ce tournant du XXe et du XXI siècles, se servent de ce vieux modèle pour dessiner, par contraste, les figures trop indécises du monde en gestation: on se raccroche à ce capitalisme " dans ses formes extrêmes " ${ }^{1}$ pour mieux s'orienter dans un présent si difficile à déchiffrer. Une périodisation courante renvoie à " plus de cinquante ans ${ }^{2}$ la disparition de ces fonctionnements dépassés ; elle doit beaucoup, consciemment ou inconsciemment, aux analyses de Karl Polanyi : la Grande Transformation, qu'il voyait s'engager dans les années 1930, aurait mis fin à la longue parenthèse, au moins séculaire, caractérisée par le triomphe de l'économie de marché ${ }^{3}$. Pour situer plus tôt, dès 1890 , le moment où "un capitalisme chasse l'autre", Charles Morazé campait ses Bourgeois conquérants du $\mathrm{XIX}^{\mathrm{e}}$ siècle dans un paysage analogue: il disait, sans en ignorer les contradictions, une aventure, intellectuelle autant que sociale, mais en tout cas individuelle, mue par l'ardeur de la concurrence, aboutissant à "proclamer la liberté d'entreprise » tout en contrôlant « ce qu'il faut bien garder d'administration publique » 4 .

Comme il arrive souvent, les bouleversements du présent sont en passe de transformer aussi notre histoire, ou du moins l'interprétation que nous en donnons. Ne sommes-nous pas en train de vivre, avec la nouvelle offensive du libéralisme économique, une sorte de " grande transformation à l'envers " ${ }^{5}$ ? Dans nos représentations, la ligne s'est peut-être brisée, qui semblait mener, par l'exigence-même de la croissance et les effets de la concentration, à la "re-socialisation » de l'économie. Le pire est que sont tenus, sur l'évolution actuelle des structures du capitalisme, les propos les plus contradictoires et selon des oscillations de plus en plus rapides, qui semblent épouser, sans aucun recul, les variations de la conjoncture. Pour n'en retenir qu'un exemple, les entreprises caractéristiques de notre temps sont-elles ces produits gigantesques de fusions, indispensables pour atteindre la taille critique à l'échelle du monde? L'entreprise du 
début du XXIe siècle n'est-elle pas plutôt cette puissance incisive, concentrée sur un métier, qui a su «externaliser» toute graisse inutile et peut ainsi mieux saisir l'innovation dans la souplesse de projets successifs? Les deux affirmations nous sont assénées en alternance quoique toujours d'une façon péremptoire.

Mais alors, la mémoire du XIX ${ }^{e}$ siècle doit-elle se limiter à utiliser un modèle univoque, simple référence rassurante pour l'exploration des évolutions actuelles? Le capitalisme du XIXe siècle était-il donc plus " pur»? Les historiens pourraient tirer un parti positif des perplexités de notre époque en reportant vers les premiers temps industriels une série de questions suscitées par l'apparition d'un nouvel esprit du capitalisme.

C'est sous ce titre que Luc Boltanski et Ève Chiapello ont présenté une magistrale étude des changements idéologiques qui ont accompagné les transformations récentes du capitalisme ${ }^{6}$. Bien sûr leur titre fait explicitement référence à l'œuvre de Max Weber et les systèmes de représentation qu'ils dégagent d'un examen comparatif des traités de management des années 1960 et 1990 sont, dans leur esprit, des moteurs puissants, comme a pu l'être l'éthique protestante ${ }^{7}$. Ils leur servent à rendre compte du succès des nouveaux modes d'organisation, y compris dans ce qu'il a de plus étonnant : la séduction qu'ils exercent auprès d'hommes et de femmes qui, dans leur majorité, ne semblent pourtant pas en éprouver personnellement les bénéfices. Mais, montrent-ils, un certain type de critique s'est trouvé désarmé, pris à contre-pied par des discours dominants qui ont, en quelque sorte, dérobé certains de ses thèmes favoris pour les utiliser à la justification du nouvel ordre.

5 La tentation de confronter cette situation à l'ordre ancien du XIX ${ }^{e}$ siècle ne vient pas seulement d'une manie professionnelle réveillée par la comparaison très implicite dont sont porteurs les auteurs de ce livre. Elle naît d'un doute quant à la nouveauté des thèmes développés dans les discours de légitimation qui sont étudiés. L'historien un peu familier du XIX' siècle n'est pas toujours aussi dépaysé qu'il s'y attend par les argumentaires d'aujourd'hui. Il est pourtant convaincu de la profondeur des bouleversements intervenus depuis «son" époque. Car, contrairement à Joseph Prudhomme, le héros d'Henri Monnier, il souhaiterait que l'histoire serve à mieux dégager ce que le présent apporte de radicalement neuf. On ne le trouve pas clairement dans la façon dont les discours récents des managers suggèrent ce que doivent être notamment la structure et l'échelle des entreprises ou le comportement de leurs acteurs. Ainsi Luc Boltanski et Ève Chiapello soulignent-ils l'appel fréquent des derniers théoriciens du management à un retour des relations personnelles dans le monde économique, invoquées contre le modèle «bureaucratique » de la période immédiatement antérieure et la rigidité inefficace de ses monstres froids. Et ils ont, bien sûr, raison de préciser qu'« il ne s'agit pas pour autant d'une tentative de retour au premier esprit du capitalisme car les entreprises sont plus grandes que jamais, les managers des professionnels et non des petits propriétaires, et la vie au travail s'inscrit dans des réseaux et non dans un cadre domestique ${ }^{8}$. Mais le plus frappant est l'énorme distance qui sépare ici discours et pratiques, en un monde où les monstres froids ne sont pas tous en voie de réchauffement. D'où la question : le travail de justification s'exerce-t-il autrement que par le maniement permanent de la contradiction? En fait d'échelle des entreprises par exemple, nous avons signalé la constance de ce grand écart. L'analyse des discours qui dégage des thèmes dominants, caractéristiques de leurs époques, doit prendre garde de ne pas les couper artificiellement des jeux d'opposition où ils s'inscrivent. 
6 Car est-il rien de plus banal que cette aptitude de beaucoup d'entrepreneurs ou de théoriciens du développement économique, à dire alternativement blanc et noir sur quelques aspects essentiels de l'activité concernée ? Ceci dans une longue durée est observable au moins à l'échelle des deux derniers siècles. Nous donnerons ici un certain nombre d'exemples de ces alternatives telles qu'elles apparaissent dans les discours des entrepreneurs du XIX siècle. Nous souhaiterions ainsi rappeler l'intérêt d'une recherche, moins pratiquée par les historiens, sur les discours et les représentations qui conditionnent ou accompagnent le développement économique. Elle pourrait tenir compte de la grille d'analyse appliquée, dans le livre cité, au nouvel esprit du capitalisme : il paraît fécond de repérer dans l'apologétique des entrepreneurs, comme l'ont fait ces deux sociologues, leurs réponses à trois principales questions dont dépend la mobilisation des populations qui les environnent : pourquoi m'engager, voire m'enthousiasmer pour une entreprise? quelles garanties, quelle sécurité m'offre-t-elle? et qu'apporte-t-elle à la société où elle s'exerce, en termes de bien commun? Mais aux trois éléments de cette problématique, combien peu de réponses univoques! Au point qu'un certain usage de la contradiction, un jeu bien dosé d'alternatives pourrait apparaître comme la quatrième dimension nécessaire de tout bon discours justificatif dans le monde des entreprises. Et le sentiment de déjà lu ou de déjà entendu qui peut saisir les historiens du XIX ${ }^{\mathrm{e}}$ siècle à la lecture de la presse économique d'aujourd'hui vient sans doute d'abord de ce qu'ils retrouvent, au-delà des contenus, un mode de fonctionnement constamment nécessaire, peut-on penser, à travers tant de bouleversements. Sa nécessité tenant, en première hypothèse, non seulement au besoin de légitimation des entrepreneurs, mais aux conditions-mêmes dans lesquelles ils exercent leur métier.

7 Nous voudrions donc montrer que, si l'on peut caractériser un ancien esprit du capitalisme, il n'est pas si facilement repérable par des traits qui l'opposeraient clairement au nouvel esprit. On ne pourra désigner ainsi qu'une certaine façon d'arbitrer un grand nombre d'alternatives, présentes depuis longtemps dans la pratique des entrepreneurs, et qui se situent inséparablement dans le dire et dans le faire. Avec un matériel restreint, nous ne proposons ici qu'une esquisse, abordant toutefois les traits par lesquels on définit le plus classiquement l'entreprise «individuelle» du XIX siècle: sa dimension modeste, familiale, patrimoniale et son caractère éminemment concurrentiel.

8 Créer ou gérer les entreprises de ces temps héroïques contraignait à des arbitrages souvent incertains. Mais les problèmes à résoudre n'ont pas tous perdu ultérieurement leur acuité dans un monde industriel qui avait changé d'échelle: nous le vérifierons d'abord à propos du contenu-même de l'activité des entrepreneurs. Pour approcher les stratégies de quelques entrepreneurs, telles qu'ils les concevaient mais aussi les donnaient à voir, faute de corpus équivalent aux collections de « management » du second $\mathrm{XX}^{\mathrm{e}}$ siècle, nous disposons de discours très disparates, plus fréquemment destinés à un usage public: réponses aux enquêtes ministérielles et parlementaires, témoignages ou autobiographies publiés du vivant de leurs auteurs. N'échappant pas à la langue de bois, ces sources sont néanmoins d'autant plus utiles qu'elles peuvent être confrontées aux pratiques des mêmes acteurs, comme le sont ou doivent l'être les déclarations des managers de l'an 2000. Mais des échantillons précieux de l'esprit de nos anciens capitalistes se surprennent mieux à la lecture de sources restées moins accessibles: correspondances, journaux, ou autres formes de discours initialement conçus à usage interne et privé. 
9 C'est le cas du « testament économique » d'André de Neuflize, négociant en laines à Sedan qui, en 1836 à la veille de sa mort, destine à son fils les réflexions entamées dans l'amertume d'une faillite ${ }^{9}$. Il peut d'abord nous intéresser par l'attention qu'il porte à la diversité des opérations qui sont réunies, dit-il, "sous la dénomination générique de commerce ». Première difficulté qui ne tracasse pas seulement les opérateurs qui ont atteint l'envergure des Neuflize: comment tenir à la fois les compétences techniques qu'exige le métier et l'aptitude au calcul spéculatif qui caractérise le capitaliste, l'ancien comme le nouveau? André de Neuflize, qui songe à la formation de sa descendance, dit la nécessité d'un double apprentissage : «calcul de tête » (base des opérations de négoce et de banque) et "jugement des yeux » (qui s'éprouve d'abord dans le «triement » des laines) permettront de fonder une réussite industrielle en contrôlant l'ensemble de ses paramètres. Il en vient pourtant à regretter le mélange des genres qui l'a empêché de concentrer son énergie sur le commerce des laines. L'incertitude réside en fait non dans la nécessité mais dans la possibilité de tenir tous les bouts de la chaîne. Confronté au même problème, Alfred Motte, grand industriel roubaisien de la seconde moitié du siècle, fit le choix de la complémentarité : il réussit une vaste intégration dans la laine et le coton en se cantonnant, en dépit d'une qualification initiale de teinturier, dans le rôle d'un « capitaliste » qui recrutait, au fur et à mesure des besoins, ceux qu'il appelait ses « artistes » ou ses « lames de Tolède ${ }^{10}$. Chacun dut surmonter l'obstacle à sa manière et celle que choisit le jeune André de Neuflize, fils du précédent et destinataire de ses recommandations, n'est pas la moins piquante : son père déconfit ne lui avait-il pas assez répété de conserver son indépendance par rapport aux banquiers? «Si on a le malheur de leur donner barre sur soi, en étant dans le cas d'en avoir un besoin trop pressant, il est bien rare qu'ils n'en abusent pas pour pressurer le négociant qui a recouru à eux, et souvent pour le renverser, lorsqu'ils ont sucé et épuisé sa substance ». Message reçu par un fils qui fonda ce qui allait devenir la banque Neuflize, Schlumberger et Mallet.

On aura reconnu ici, dans sa version la plus globale, une hésitation centrale du monde des entreprises pris entre une exigence "généraliste » (éviter la dépendance et réduire les coûts en réunissant l'ensemble des opérations nécessaires) et la recherche de l'excellence dans un ou quelques métiers. Les exemples en abondent, dès qu'une industrie connaît un certain développement, à des époques que le discours économique d'aujourd'hui aurait tendance à considérer comme préhistoriques. L'alternative se développe dans une série de questions qui, pour l'essentiel, restent sans réponse, tant la contradiction est forte entre, d'un côté, les demandes du marché et la recherche du profit, de l'autre le besoin de limiter la masse de fonds immobilisés et d'accélérer leur rotation. Rien, on va le voir, qui dépayse l'observateur d'aujourd'hui dans les discours anciens consacrés soit à la spécialisation des produits, soit à l'étendue des stocks.

11 Le dilemme spécialité/variété est bien énoncé chez un autre industriel de la laine, voisin des Neuflize, le Verviétois Henri Peltzer, qui a rédigé vers 1845 un précieux Guide sur la gestion d'une fabrique de tissage de laine ${ }^{11}$. Il y dit, à quelques pages de distance, les séductions de la variété... «La variété séduit la pratique, facilite et augmente la vente, mais pour cela [...] il faut s'y prendre à l'avance et savoir son métier [...]»; mais il doit le reconnaître en même temps : «[...] Nos concurrents qui habituellement ne font qu'un genre [...] ont bon pied et bon œil »; jusqu'à conclure, mais très provisoirement : «le fabriquant doit se faire une réputation pour une spécialité, cela facilite sa vente et lui facilite la perfection. Nous en sommes venus à ne faire qu'étoffes et noir, par l'inconstance on écarte naturellement une vente régulière ». 
12 Économies d'échelle, permises par les longues séries ou/et réponse aux défis toujours renouvelés de la mode, le problème est bien connu et sa permanente actualité déborde infiniment le cas des industries textiles. On a su en donner des analyses théoriques, distinguant des "mondes de production»: ceux qui se consacrent à des "produits standards » s'opposant par exemple aux entreprises de "produits dédiés » (dédiés à une clientèle repérée, qui permet de développer, dans des qualités supérieures, une industrie de la variété) ${ }^{12}$. Et cette opposition n'est pas sans évoquer la comparaison classique des deux grands foyers de la première industrialisation : la forte spécialisation de l'industrie anglaise versus les productions plus variées et plus « haut de gamme » des Français. Mais il est probable que, au fur à mesure que progressera notre familiarité avec l'« esprit » de ces premières générations d'industriels, nous constaterons, comme chez Peltzer, que l'incertitude et la perplexité sont plus fréquents que les choix clairs correspondant à ces typologies : la plupart apportent à la question des réponses successives, ne serait-ce que parce que la conjoncture ou le changement technique transforment leur évaluation des marchés. On sait par exemple comment l'usage élargi du métier jacquard permit à un certain nombre de places textiles de sortir de la crise de 1830 en trouvant le chemin de la " nouveauté », c'est-à-dire en limitant le coût d'une économie de la variété. «Il faut moins varier le dessin que les nuances ", écrivait aussi Peltzer.

Pour ce qui est des stocks de matières premières ou de produits, on pourra être surpris de constater que, si la notion de "flux tendu» semble aujourd'hui une composante importante du nouvel esprit industriel, elle n'est pas si étrangère à nos anciens entrepreneurs. Il y a souvent en eux à la fois du négociant séduit par le jeu sur les variations de cours et les tarifs de fret, et du bon manufacturier dont l'idéal, comme celui de Peltzer, est une fabrique "au clair »: où rien ne s'accumule à aucune étape de la fabrication. Les raisons en sont d'abord techniques : « le vieux charbon perd de la qualité [...]. Les chaînes collées sont plus tôt attaquées de vers que les chaînes qui ne le sont pas [...]. Il ne faut absolument pas de chaîne, ni ourdie, ni collée d'avance ». Mais cet attachement à un just in time du XIX siècle tient d'abord à une analyse commerciale et financière : « une trop grande quantité de produits fabriqués sont une plaie, un cancer rongeur ». Outre le désir de limiter l'immobilisation des fonds, c'est affaire d'image : « un fabricant doit toujours passer pour ne pas avoir de marchandise, il doit autant que possible faire croire qu'il en est dépourvu car l'acheteur accourt où il croit que les acheteurs affluent et où il sait qu'il n'y a pas de vieille marchandise».

L'opportunité de constituer des stocks de matière première est un sujet plus débattu, peut-on constater à la lecture des réflexions d'un Peltzer ou de la correspondance des Seydoux du Cateau ${ }^{13}$. Vers 1835, André de Neuflize voyait l'avenir dans la fourniture, à chaque utilisateur de laine, des variétés qu'il lui était le plus difficile de trouver. Mais pour jouer sur toute la gamme de qualités et de prix disponible en France, Allemagne, Espagne, Portugal, un négociant habile pouvait ne se fournir lui-même à la source qu'au fur et à mesure des demandes. Avec l'éloignement et la centralisation des approvisionnements, à Londres puis à Sydney et Melbourne, et l'importance prise par les grandes ventes qui s'y organisaient, il devint plus difficile de contenir ses achats dans les limites d'une consommation ou d'une vente assurées et on fut tenté de s'exposer davantage aux immobilisations et aux risques des variations de cours sur les stocks. Il est vrai que, à partir des années 1850, l'envoi d'acheteurs par les grandes maisons de négoce vers l'Australie, puis l'Argentine ou l'Afrique du Sud put rétablir la possibilité d'une fourniture directement liée à la commande. Jusqu'au moment où ces acheteurs eux- 
mêmes, comme le montre l'exemple de l'un d'entre eux en Afrique du Sud, déplacèrent vers les lieux lointains d'approvisionnement la même alternative : ils combinèrent l'achat sur commande avec la constitution d'assortiments ouverts à la variété des demandes potentielles ${ }^{14}$.

Autant de choix laborieux, de contradictions qui, jusqu'ici, tiennent à l'objet-même de ces entreprises et à la délimitation de leurs activités. Ce ne sont que quelques notations qui suffisent pourtant à convaincre, si nécessaire, que, pour exercer leur art à une échelle relativement modeste, nos entrepreneurs n'en ont pas moins affronté des contradictions qu'on peut retrouver tout au long de l'histoire de l'industrie moderne. Mais la dimension familiale, caractéristique de la majorité des entreprises du XIX siècle, ne lui confère-t-elle pas des traits à la fois plus univoques et plus spécifiques? À l'examen des mêmes sources et de quelques autres, il nous faut bien admettre que la plupart des évocations récentes de ce passé doivent tout à un éloignement qui simplifie l'épure.

Non que la nature familiale de ces firmes, de ces ateliers, de ces premières usines soit contestable. L'entreprise est familiale en fait, l'écrasante majorité des premières sociétés le sont en droit, adoptant en France le statut des sociétés en nom collectif pour réunir diverses combinaisons de parenté. Et le procès sans cesse repris qui, au $\mathrm{XX}^{\mathrm{e}}$ siècle, a incriminé cet enracinement, cet ancrage dans l'archaïsme, s'alimente de nombreux arguments. Borné par l'horizon familial, l'entrepreneur est retenu devant les changements nécessaires: il aurait besoin d'investir de nouveaux fonds, mais il en est empêché par un cercle familial trop frileux, par le désir de garder le contrôle, de ne pas voir des étrangers mettre le nez dans ses comptes. La gestion quotidienne, que pourrait réformer un gérant ardent et inventif, est entre les mains de fratries trop nombreuses et tel créateur talentueux doit supporter un frère, un cousin, pire un beau-frère paresseux et dissipateur...

17 Tout ceci a sa part de vérité mais, vu de plus près, devrait nous conduire à rompre avec l'image de l'individu-aventurier qui domine encore l'historiographie; et de ce fait à relativiser ce que cet objet ancien a de spécifique : aventure individuelle? Tout indique les souffrances de l'individu-entre-preneur qui a une claire conscience de ce que la famille est la meilleure et la pire des choses ! Et il ne manque pas d'opérateurs clairvoyants pour imaginer des solutions, longtemps avant Alfred Chandler Junior ${ }^{15}$.

On sait très tôt la lourdeur des familles : « il faudrait », suggérait Henri Peltzer vers 1845, " nous réunir sérieusement en conseil, ainsi que cela se fait en toute administration; nous sommes trop nombreux et par là nous éprouvons trop de tiraillements [...]. Aujourd'hui quand j'ai répété 100 fois la même chose, c'est comme si j'avais chanté ». Et, quelques temps après, en 1877, Alfred Motte dit crûment les choses à son ami lyonnais, Joseph Gillet, lorsqu'il lui faut soutenir un frère et un beau-frère défaillants: "nous ne gagnerons jamais trop d'argent parce que nous serons toujours entourés de gens qui n'en auront pas assez ${ }^{16}$.

19 Mais l'auto-analyse peut aller plus loin, jusqu'à la remise en cause de la dispersion des intérêts, généralement considérée comme l'effet nécessaire de la nature familiale des entreprises. Nous en trouvons un témoignage dès le milieu du siècle chez un auteur qui nous permet d'étendre notre matériel au-delà des affaires textiles où nous nous sommes cantonnés jusqu'ici : Gustave Goldenberg, grand quincaillier alsacien (1805-1871) a publié en 1856 une réflexion prospective qu'il a intitulée tout simplement: De l'avenir de notre société ${ }^{17}$. Choqué comme beaucoup d'entrepreneurs par la révolution de 1848, dont il comprend bien le lien avec la crise conjoncturelle, il préconise de façon très argumentée 
une concentration de l'industrie : les chemins de fer, explique-t-il, ont familiarisé « toutes les classes de la société avec les grandes associations industrielles, commerciales et agricoles ». Il faut donc réunir, fusionner les établissements existants. Il en attend une efficace correction des cycles! «Plus de régularité dans la production et plus de régularité dans la vente ». Il promet, avant la lettre, des économies d'échelle, passant notamment par l'achat groupé des matières premières. Et il va jusqu'à dessiner un modèle de gestion par «managers ", que ne récuserait pas Chandler ; le greffant, il est vrai, sur cette pratique disparue avec l'ancien esprit du capitalisme, la retraite précoce: l'entrepreneur pourra se retirer en trouvant un gérant parmi les nouveaux associés, « soit même parmi des étrangers qui présenteraient des garanties de moralité et de capacité ».

Cependant, du dire au faire il y a souvent loin. Et l'intérêt du propos de Gustave Goldenberg est redoublé par la connaissance que nous avons, par ailleurs, de sa pratique, qui paraît toute différente. Son exemple témoigne ainsi de la parfaite ambiguïté du modèle de l'entreprise familiale : s'il fit d'abord grossir son industrie de quincaillerie en absorbant quelques autres usines, la réorganisation qu'il opéra en 1863 consista à replacer l'ensemble sous le contrôle exclusif de sa famille ${ }^{18}$. Il était apparemment conscient aussi de ce qu'une famille peut apporter, au développement d'une industrie, des ressources considérables dont les générations suivantes ne se passeront pas si facilement.

C'est que, malgré ses limites ou ses tares, la famille représente aussi pour l'individuentrepreneur la principale chance de socialisation et d'inscription dans la durée. Nous savons depuis longtemps que les dimensions ordinairement modestes où se cantonne l'entreprise du XIX siècle peuvent correspondre à un choix rationnel ${ }^{19}$. L'analyse des actes de sociétés permet de préciser les voies par lesquelles on mobilise et l'on rassure les acteurs de ces milieux d'abord restreints. En effet ces contrats, sous la formule du nom collectif en particulier, servent à élargir et à consolider les virtualités de la relation de parenté ou d'alliance. Ils servent à réunir des capitaux, mais aussi des talents et des énergies complémentaires; et ils fournissent ou redoublent les garanties dont les collaborateurs ne peuvent se passer : à court terme, les avantages d'un système de crédit solidaire et ceux d'une confidentialité que les firmes du $\mathrm{XX}^{\mathrm{e}}$ siècle peineront à entretenir par d'autres voies; ils promettent pour l'avenir, avec la transmission du flambeau, continuité du projet et entretien des savoir-faire (on ne parlait pas alors de «culture d'entreprise »). On comprend par là le succès des sociétés familiales, au-delà du milieu textile où l'urgence de collecter des fonds importants est moins précocement ressentie, succès prolongé longtemps après le changement législatif de 1867 qui rendait pourtant accessible le statut de société anonyme.

De ce fait l'entreprise familiale a pu accueillir une bonne partie de la croissance, voire de la concentration de la seconde moitié du XIX siècle: l'exemple de la quincaillerie Goldenberg pourrait être corroboré par quantité d'autres, dans le Nord notamment. Et aussi en Angleterre où l'on a pu mettre en évidence, jusqu'aux années 1930 au moins, la capacité de firmes familiales produisant de l'alimentation de qualité - branded goods comme Cadbury et Rowntree à se développer sans se plier au modèle "managerial ", quitte à en intégrer les éléments jugés pertinents ${ }^{20}$.

Derrière ces contradictions du modèle familial, l'analyse gagnerait en fait à repérer les variations sur un thème qui travaille encore plus profond, s'il est possible, l'esprit de nos anciens capitalistes : celui de la propriété. Car nous touchons alors à ce que Max Weber recherchait dans l'«esprit du capitalisme»: au-delà des utilités, une éthique, l'affirmation d'un principe, d'un summum bonum dont la transcendance s'impose aux 
milieux d'affaires comme à l'ensemble de la société ${ }^{21}$. Il est d'autant plus important de repérer les obstacles intellectuels auxquels les entrepreneurs se heurtent sur ce sujet. Tous propriétaires, nos entrepreneurs ou aspirant à le devenir toujours davantage ? Sans doute mais, dans la confusion qui règne souvent entre entreprise et patrimoine familial, il n'est pas si facile de s'orienter.

Pas besoin de longues démonstrations pour rappeler que la propriété est à la fois fin et moyen pour l'entrepreneur, l'horizon qui mobilise, le levier qui opère, le statut qui rassure. Elle donne tout son sens au travail, autre valeur souveraine : on s'épuise à la tâche pour accumuler et transmettre aux générations suivantes non seulement une richesse accrue, mais aussi un exemple qui contribuera à maintenir l'équilibre social; et la motivation du propriétaire, ce ressort décisif, sert d'argument imparable contre la perspective des directions salariées, de ce qui sera l'intrusion des managers: Thiers déplorait dès 1848 la menace de voir substituer « des administrateurs à des propriétaires, c'est-à-dire un ressort très faible à un ressort très énergique ». Récusant d'avance l'opinion d'un Goldenberg, il appelait « les filateurs de coton et de lin, les fabricants de toile peinte, les fabricants de lainage de Mulhouse, de Saint-Quentin, de Lille, de Rouen, d'Amiens " à témoigner: "La nécessité de l'œil du maitre est un vieil adage qui est et restera éternellement vrai. Il faut dans les affaires privées la vigilance, l'attention passionnée de l'intérêt individuel, et non le zèle affaibli de l'intérêt collectif » ${ }^{22}$.

Négociants et industriels du XIX siècle sont néanmoins bien conscients de ce que leur patrimoine est menacé, que la condition de propriétaire-entrepreneur expose en même temps qu'elle assure. Aventuriers modernes, mais pas tous téméraires, ils souhaiteraient, bien naturellement, minimiser les risques. Mais les statuts de leurs associations et l'ensemble de leurs documents commerciaux traduisent deux volontés largement contradictoires: chacun souhaite savoir tout de ses partenaires, associés, clients, fournisseurs, et vérifier qu'ils sont complètement responsables sur leurs biens, qu'ils engagent sans réserve leur nom ou leur raison sociale; en revanche chacun rêve de ne pas trop s'exposer lui-même. C'est tout le fond d'un système qui a été décrit pour LilleRoubaix-Tourcoing au milieu du XIXe siècle: une constellation de sociétés liées par la parenté, exerçant des activités complémentaires (négoce, industrie à forfait, industrie à façon) mais cloisonnées par des sortes de portes coupe-feu ${ }^{23}$.

Non qu'on ignore un remède que la législation rendra bientôt plus praticable: la responsabilité limitée, mais toute la difficulté vient de ce que cette solution expose la propriété d'une autre manière. Gustave Goldenberg était capable dès 1856 de concevoir une transformation de la fortune des «bons et honorables fabricants » : elle deviendrait mobilière, en actions qui pourraient se placer facilement « et même avec bénéfice ». Mais, on l'a vu, il ne suivit pas pour son propre compte la voie vantée, dont l'exemple était fourni par les compagnies de chemin de fer : comme la plupart des industriels, il mesurait sans doute le risque de perdre en contrôle de son entreprise ce qu'il gagnait en surface financière. Entre les écueils, chaque entrepreneur réglait son cap à sa manière et tous ne suivaient pas les plus réticents devant l'évolution du financement industriel. Ceux-ci abondent dans le Nord textile, bien représentés par la fameuse répartie du linier lillois Scrive en 1838 ; interrogé par le ministère sur l'éventualité d'un passage à la société par actions, il rétorquait : «nous sommes des fabricants sérieux; nous ne vivons pas sur des actions au porteur, nous ne voulons pas jeter aux chiens un nom qui déjà compte honorablement parmi les industriels ${ }^{24}$. Sa réponse mêlait sans doute à un désir de conserver crédit, réputation, chalandise, la peur d'introduire en fait des loups dans sa 
bergerie et de devoir rendre des comptes. Les générations suivantes, sans abandonner ces préoccupations, imagineraient des expédients intéressants. Ainsi des compagnies houillères, qui réussirent parfois à récolter des fonds sans rompre avec la bonne habitude de régler les choses dans un cercle restreint. On peut du moins l'inférer de ce que déclarera candidement en 1901 un administrateur de l'une de ces compagnies. Elles avaient conservé le statut de sociétés civiles et se contentaient jusqu'alors de drainer des fonds (capital et obligations) à la Bourse de Lille, lorsqu'il fut question de chercher l'admission à la cote parisienne; objection : coter à Paris contraindrait à publier des résultats! ${ }^{25}$

Que mettre de son patrimoine dans l'entreprise, et quelle part y admettre de celui d'autrui? Mais aussi quel usage faire de ses fonds hors de l'usine ou du négoce? Questions simples à énoncer, que les acteurs du XIX siècle peinent à résoudre. Ainsi, à en croire Henri Peltzer, il ne suffit pas d'accumuler autant qu'on le peut : «Il faut toujours conserver portefeuille assez garni pour profiter d'une bonne occasion d'acheter de la laine dans un moment critique ", mais attention: «lorsqu'une maison a trop de fonds, elle les laisse chômer, souvent mal à propos, bien des maisons ont succombé par là ». On sait comment beaucoup se débrouillent alors de cette alternative : en fondant leur crédit sur des biens-fonds, mobilisables au besoin ${ }^{26}$. Mais en revanche, André de Neuflize voyait dans cette immobilisation une cause majeure de sa faillite, comme des revers retentissants subis par Ternaux puis par Laffitte : certes ils n'auraient pas dû se mêler de politique ${ }^{27}$ mais, plus généralement, expliquait-il, ils auraient dû réserver à leur commerce tout leur temps et tous leurs fonds. «Mes huit trisaïeuls, mes quatre bisaïeuls, mes deux aïeuls étaient exclusivement manufacturiers et négociants. Ils avaient presque toute leur fortune dans leur commerce ou en valeurs réalisables à l'instant [...]. C'est que le commerce n'admet pas que l'on soit simultanément propriétaire foncier, si ce n'est de propriétés à son usage ou pour votre délassement, ou encore toujours dans une proportion minime, comparativement avec les capitaux roulants qui lui sont consacrés » ${ }^{28}$.

Allez donc savoir ! Comment faire « travailler » exactement l'argent dont on dispose sans en manquer ni en laisser « chômer » ? Et comment assurer la croissance d'une entreprise sans faire courir à sa fortune personnelle les aventures de l'endettement, sans l'ouvrir dangereusement au public ? Il faut bien admettre que, en dépit d'un complet changement d'échelle, l'histoire industrielle des deux derniers siècles n'a pas vu trancher ces questions entêtantes de façon définitive. Sans doute trouvera-t-on de moins en moins d'entrepreneurs pour se vanter de ne devoir rien à personne. Revenant sur les succès de son père au centenaire de sa naissance en 1927, Eugène Motte (fils d'Alfred) fait désormais de l'endettement vertu. Cette pratique figure parmi les « secrets » qu'il dévoile dans un grand discours public: "le secret, c'est d'avoir le sens de l'honneur de sa signature et d'avoir pu emprunter des sommes considérables pour l'époque à ses parents, à ses proches [...], à la ville entière, et surtout aux banquiers et c'est encore aujourd'hui notre cas. [...] Trois fois il fut à jour, quatre fois il recontracta des emprunts et signa des reconnaissances de dettes à termes échelonnés et aventura le tout dans de nouvelles créations. Chaque fois qu'il gagnait 1000 francs, il en empruntait autant pour en dépenser $3000 »{ }^{29}$. Que le crédit s'éprouve désormais par l'étendue de la dette, et non plus par la « mise à jour » qui garantit la puissance propre de l'entrepreneur, nous serions tentés de placer là une rupture décisive, ouvrant l'ère du nouveau capitalisme. De ce stade à la shareholder value, à un crédit démontré par la valeur boursière, il n'y aurait 
qu'un pas. En cette hypothèse, la fin du XIX ${ }^{\mathrm{e}}$ siècle aurait vu disparaître définitivement des comportements timorés. Le pouvoir économique ne se savoure-t-il pas aujourd'hui en réunissant ses actionnaires par milliers au Palais des Congrès ou dans la Cour Carrée du Louvre?

Ne perdons cependant pas mémoire d'alternatives anciennes, qui révèlent des contraintes ou des contradictions dont la disparition reste aujourd'hui à démontrer. Il est sans doute difficile d'apprécier la portée de retournements qui peuvent apparaître tout conjoncturels : ainsi le mouvement qui a conduit, en l'an 2000, 150 sociétés américaines et quelques sociétés françaises à quitter volontairement la Bourse ${ }^{30}$. Mais accorde-t-on assez d'attention aux discours récurrents des grands managers sur « les entreprises sans usine ", ou autres formules traduisant un recul devant l'importance des capitaux immobilisés? Tel dirigeant de grand groupe mondialisé parle de "sortir de la matière " ou, encore mieux, de «se dégager de la propriété ${ }^{31}$. Il faut évidemment faire la part d'une rhétorique, correspondant bien aux analyses de Boltanski et Chiapello et qui, en vantant la mobilité, le «nomadisme » des nouveaux entrepreneurs (délivrés des grandes machines bureaucratiques...), jette un voile de fumée sur une énorme accumulation de capitaux. Reste la réalité d'une préoccupation: que ces capitaux soient toujours disponibles, convertibles, que les directions jouissent d'une complète liberté opérationnelle. Ce qui n'est pas facilement compatible avec le pouvoir dévolu aux actionnaires, ou dépend de l'habileté des administrateurs à leur faire prendre des vessies pour des lanternes, à arranger les bilans ou, comme le disent joliment certains dirigeants d'entreprises, «à faire de la peinture ».

30 Autrement dit, si tout a changé et si les multinationales, " opéables » ${ }^{32}$ de surcroît, n'ont apparemment rien à voir avec nos vieilles industries en nom collectif, ne pouvons-nous repérer, de part et d'autre et au long de deux siècles, les fluctuations et les retours d'un discours sur la propriété ? Il doit sans cesse ruser avec des évidences non interrogées, aussi bien pour répondre à ce que la gestion courante comporte d'exigences contradictoires que pour légitimer la place de l'entreprise au regard des valeurs de la société. Justifier qu'on entre ou qu'on sorte de la Bourse, faire valoir l'héroïsme individuel d'un pionnier ou vanter les bienfaits qu'une industrie dispense à un monde qu'on suppose peuplé massivement d'actionnaires, ce sont autant de figures réversibles. Nous ne cessons d'être exposés à des retournements, qui démentiront bien des analyses péremptoires des économistes, et disqualifieront aussi les périodisations trop sommaires des historiens.

31 La définition du premier capitalisme industriel comme capitalisme de concurrence généralisée devrait mieux résister au réexamen qui accompagne nécessairement la marche de l'histoire et le renouvellement des questions qu'on lui pose. La concurrence est au cœur du « credo libéral » qui, selon Karl Polanyi, s'est imposé dans les années 1830. Et, dans l'ordre des représentations, n'est-elle pas le signe le plus manifeste de cet « esprit de croisade passionnée » qui, au cœur du XIX ${ }^{e}$ siècle, détermine toute la législation, met hors la loi ententes et coalitions, imprègne la majorité des discours publics et privés ? ${ }^{33}$

À côté de tous les éléments qui valident cette description, il vaudrait cependant la peine de s'interroger encore sur les raisons qui, préludant dès la fin du XIX ${ }^{\mathrm{e}}$ siècle à la Grande Transformation des années 1930, ont conduit à tempérer le principe de concurrence et à en restreindre sérieusement l'application. Le débat, rouvert ces derniers temps pour la énième fois, oppose, on le sait, des libéraux affirmant que, si le système n'a pas fonctionné jusqu'à présent comme il le promettait, c'est faute d'avoir été complètement installé ; et des critiques du laissez-faire prêts à attribuer le changement de cap, comme le 
faisait Polanyi, à « la manifestation des faiblesses et des périls inhérents à un système de marché autorégulateur $"{ }^{34}$. L'intérêt de la recherche que nous préconisons serait d'aller regarder plus en amont tout ce qu'en disent et ce qu'en font les premiers intéressés, les entrepreneurs eux-mêmes. Peut-être apparaîtra-t-il au moins que, si la concurrence n'a guère été pratiquée à la Boisguillebert ${ }^{35}$ ou à la Bastiat ${ }^{36}$ (ce qui rendait impossible la vérification du bien fondé des Harmonies économiques), c'est que, d'emblée, les acteurs doutaient de leur chance de s'enrichir en l'affrontant sans précautions.

Pas question pourtant de négliger les éléments structurels qui sous-tendent le développement de la concurrence : la dispersion des intérêts n'a rien d'un fantasme et la première industrialisation concerne un semis de petites entreprises soucieuses de tirer leur épingle du jeu ; en face, du côté de la demande, de profonds changements ont affecté la stratification sociale, les pouvoirs d'achat, les modèles de consommation. Et, bien avant le temps du consumérisme, a pu s'exprimer dans les clientèles une volonté forte d'animation des échanges et de régularisation des prix qu'on peut attendre de la libre concurrence. Mais, avec la perte de flexibilité de la production, les entrepreneurs doivent, dans les mêmes années du XIX siècle, s'adapter à l'instabilité de la demande jusqu'au moment où ils s'efforceront de "conformer le marché aux nécessités de leur production ${ }^{37}$.

Dans ces conditions, nos praticiens peuvent-ils s'accommoder de la concurrence comme les poissons de l'eau ? À les entendre et à les observer, il faut pour le moins admettre que meilleure qualité et prix plus bas ne constituent que deux moyens parmi d'autres de conquérir les marchés et de prospérer. Et d'abord pour cette raison d'évidence que les entrepreneurs évoluent dans un monde de transactions - ponctuelles - et de relations - plus durables - où il leur faut sans cesse se mesurer ou négocier avec des concurrentspartenaires, des amis-ennemis. André de Neuflize résumait bonnement ce problème en 1836 : «il est impolitique de se mettre en rivalité avec des personnes de la bienveillance desquelles on a besoin ». Le quotidien les voit effectivement se combattre et échanger de bons procédés, se visiter courtoisement et, à défaut de s'espionner, exercer les uns chez les autres, avant la lettre, une sorte de "veille technologique ». Et périodiquement, les plus clairvoyants ou les plus solides en viennent à crier casse-cou.

Un bon exemple en est fourni dans les registres des Houillères et fonderies de l'Aveyron. La correspondance échangée en 1841 entre Cabrol, qui dirige la production à Decazeville, et le conseil de la compagnie, qui siège à Paris, ne témoigne d'aucune sympathie pour le capitalisme de concurrence. Pratiquer les affaires dans cet « esprit » ne permettrait ni de faire baisser le prix du bois, ni de faire monter le prix des rails, deux objectifs qui s'imposent pourtant. Le premier implique l'organisation de tous les maîtres de forges locaux : «Au nombre de sept à huit seulement, ils se laissent faire la loi par plusieurs centaines de propriétaires, ce qui me parait absurde puisqu'il leur serait si facile de s'entendre. Decazeville serait l'âme de cette association qui, si elle peut se réaliser, laisserait aux maîtres de forges actuels le soin de produire la fonte pour nous avec des bénéfices basés principalement sur la réduction du prix du bois, en nous garantissant, bien entendu, un approvisionnement certain à des prix modérés ${ }^{38}$.

Quant aux grandes affaires, les commandes de rails, elles se traitent à Paris. En février 1841, une adjudication de rails pour le chemin de fer du Nord nous fournit un bon exemple de compétition, telle qu'on la pratique alors dans la sidérurgie. Les dirigeants parisiens racontent à Cabrol comment, en accord avec « M. Scheneider », ils s'efforcent de convaincre les autres candidats (forges du Nord, forges d'Alès et « M. Windel d'Ayanges ») 
d'éviter les malheurs de la concurrence: «Nous avons cherché à nous mettre d'accord pour maintenir les prix dans des proportions raisonnables, écrivent-ils le 16 février, et nous n'avons pu, jusqu'ici, y parvenir. Hier nous devions donc nous présenter dans toute la rigueur d'une vive concurrence, et nous le regrettions beaucoup ». Le 19, les maîtres de forges du Nord apparaissent toujours coriaces: «Fallût-il faire un sacrifice sur les quantités à obtenir dans les chemins du Nord, nous pensons que ce sera bien agir pour conserver les prix au chiffre où ils sont aujourd'hui... ».

C'est le 22 enfin que l'adjudication a lieu au ministère des travaux publics et la bonne nouvelle part le lendemain pour Decazeville: «Le chemin de Lille à la frontière de Belgique, comprenant une fourniture de 13600 rails nous a été adjugé collectivement avec Le Creuzot, au prix de $405 \mathrm{f}$ la tonne. Cette quantité devra être livrée dans la proportion de 8/18 pour Le Creuzot et 10/18 pour Decazeville. Dans le but de nous assurer une adjudication, nous avons dû prendre quelques obligations dont vous voudrez bien prendre note : ainsi, nous céderons 300 t à Hayanges sur le marché de 2000 qui nous a été proposé par la compagnie de Rouen et sur l'adjudication de $1000 \mathrm{t}$ faite par la même compagnie au profit du Creuzot ».

La suite du courrier n'est pas moins intéressante: la compagnie de Decazeville et les Schneider n'ont pas seulement partagé, hors adjudication, les marchés du Nord et de Rouen avec les Wendel. Ils ont payé le retrait des forges du Nord, ne dépensant ainsi évidemment qu'une part de ce qu'ils ont gagné en remplaçant l'adjudication concurrentielle par une entente: «Enfin nous avons pris avec Le Creuzot l'engagement de bonifier $5 \mathrm{f}$ par tonne aux forges du Nord, la somme résultant de cet engagement devant être payée à un an et deux ans de la date de l'approbation de l'adjudication » ${ }^{39}$.

On pourra après tout juger que le combat avait été chaud, même s'il s'était déroulé en violation complète de l'article $419 \mathrm{du}$ Code pénal qui prohibait les ententes. Nous savons aujourd'hui, par l'exemple de la compétition sportive, que le meilleur, celui qui gagne, a pu utiliser des procédés longtemps considérés comme non canoniques. Et, dans les deux cas, celui du sport, celui des affaires, il ne manquait, il ne manque pas de pratiquants que l'émulation anime et conduit à de grandes actions. Irions-nous jusqu'à dire que «les Français n'ont jamais douté des vertus de la concurrence " ${ }^{40}$ ? C'est bien sûr affaire de conjoncture et de position relative: après la crise de 1846-1848, Gustave Goldenberg souhaite clairement qu'il soit mis fin aux «chômages résultant d'une concurrence effrénée, irréfléchie et désastreuse pour chacun ». En 1860, après la conclusion du traité franco-anglais qui ouvre le champ de la concurrence entre les entreprises des deux pays, la très large consultation organisée par les autorités montre au moins que tous les industriels ne sont pas mécontents: la concurrence sourit à certains acheteurs, par exemple aux compagnies de chemins de fer acheteuses de rails, pour des raisons compréhensibles; ou au baron Seillière, premier acheteur de laine brute du pays et qui a la fourniture de tout le drap de troupe et des couvertures de campement pour le gouvernement ${ }^{41}$. Mais l'exemple de ceux qui n'ont rien à craindre de la concurrence ne suffit pas à caractériser l'esprit du capitalisme dans cette phase! Tout au plus pourra-t-on observer que, pour des raisons politiques et idéologiques, les ententes et coalitions restent longtemps discrètes ou secrètes. Il faudra encore un demi-siècle pour que les tribunaux absolvent des ententes manifestes et jugent, comme le tribunal de Saint Étienne en 1911 qu'« un groupement destiné à l'unification des prix de fabrication pour en empêcher l'avilissement ne porte pas atteinte à la liberté du commerce et de l'industrie et ne fait pas obstacle à la libre concurrence " ${ }^{42}$. Mais là ne s'arrêtent pas les 
incessantes contradictions des discours sur cet objet jusqu'au temps de Microsoft et des « ententes concurrentielles » du XXI ${ }^{e}$ siècle. rapprochements ou en dessinant de telles perspectives cavalières, nous n'avions nullement l'intention de minimiser l'évidente profondeur des changements intervenus depuis ces temps lointains, dans l'échelle et dans la nature-même du capitalisme. Mais était-il tout à fait inutile de rappeler à quel point la forme d'organisation du monde de la production et de l'échange que nous désignons ainsi, déborde sans cesse les modèles où l'on essaye de l'enfermer? Sans cette mobilité incessante, comment comprendre son caractère jusqu'à présent insubmersible? D'où ce truisme : que le succès dépend d'une bonne navigation et qu'aucune formule - fût-elle dans l'air du temps - ne peut le garantir; puis une hypothèse qui concerne cette fois les discours: qu'un registre d'alternatives disponibles constitue depuis au moins deux siècles le matériel utilisé pour cette navigation incertaine. Il serait intéressant de lire de nouvelles archives avec cette curiosité.

41 De ces instruments de bord nous n'avons présenté ici que quelques exemples. Nous ne sommes pas revenus sur le thème, contradictoire entre tous, de la relation à l'État et aux pouvoirs publics ${ }^{43}$. Nous n'avons guère abordé les alternatives que prétendent résoudre les trois dogmes classiques du credo libéral au XIX ${ }^{\mathrm{e}}$ siècle: sur le prix du travail (le marché), la régulation de la monnaie (l'étalon or), l'organisation du commerce mondial (le libre-échange). À défaut de dresser l'inventaire des discours usuels, nous souhaitions seulement ici insister sur leur caractère antinomique et répétitif.

Quiconque poursuivra l'analyse observera que les discours de l'entreprise ne remplissent pas tous la même fonction. Ils expriment d'abord la difficulté des tâches à accomplir et traduisent la perplexité des acteurs devant l'ambiguïté des situations. D'autres offrent davantage l'aspect de discours de légitimation, sans qu'il soit toujours facile de faire la part de ce qui n'est destiné qu'à l'usage externe. Les clichés répandus sur la concurrence entreraient bien dans cette dernière catégorie et contribuent, on le sait, à résoudre la «question sociale ", en l'éludant. Certains propos relèvent moins évidemment de l'« hypocrisie bourgeoise ", d'autant moins qu'ils semblent exprimer de vrais blocages intellectuels ou affectifs, peu favorables au développement des affaires... du moins en une conjoncture donnée; des discours analogues s'avéreront plus opératoires en d'autres circonstances, ayant résisté à l'usure du temps.

Restera surtout à dire s'il est possible de caractériser un ancien esprit du capitalisme ! Aux quelques pages critiques qui précèdent, on pourrait objecter avec raison que les arbres ne doivent pas cacher la forêt : d'un siècle à l'autre les destinataires ont changé, des discours semblables ont pris un sens nouveau parce que les entrepreneurs n'ont plus à s'imposer à une société rurale et rentière où ils étaient minoritaires. " Ma mission est de créer des établissements qui ne rapportent qu'un peu plus de six pour cent, intérêt et amortissements compris. Même dans ces conditions, je crois qu'on fait bien de placer ses propres capitaux dans l'industrie. Vous avez un rendement égal aux valeurs qui produisent le plus [...] enfin vous avez, bienfait inappréciable, l'obligation quotidienne du travail, à peine de voir tout s'effondrer si vous négligez la surveillance de vos usines » ${ }^{44}$. Pour l'industriel qui s'exprimait ainsi en 1880, l'entreprise devait encore faire ses 
preuves; elle n'était qu'une des activités possibles et ne le cachait pas, souhaitant seulement faire école par de beaux exemples de vertus. Il faudra quelques années de plus pour que l'esprit du capitalisme implique une mobilisation générale de la société. C'est tout le mérite de Max Weber de l'avoir pressenti dès l'entrée du XX siècle: "Chacun trouve aujourd'hui en naissant l'économie capitaliste établie comme un immense cosmos, un habitacle dans lequel il doit vivre et auquel il ne peut rien changer - du moins en tant qu'individu " ${ }^{45}$. Mais nous ne connaissons la suite que jusqu'à ce soir : aucun de nous, de ceux qui font l'histoire ou de ceux qui la racontent, ne peut parier sur la durée d'une adhésion qui est ancrée dans l'implicite. Évidemment nous ne sommes plus aux premiers temps de l'industrialisation où trop de biens, tristement matériels, étaient exposés à la convoitise de ceux qui en étaient privés. "Les négociants, écrivait Saint-Simon, sont, de tous les propriétaires, les plus intéressés à l'établissement du nouveau système de morale : car ils sont ceux dont les propriétés sont les plus exposées au pillage ${ }^{46}$. La nouvelle économie promet beaucoup. Dès que tous les biens seront dématérialisés, ou dès que cessera de se poser la question de leur répartition, la page de l'ancien capitalisme sera définitivement tournée.

\section{NOTES}

1.. Jean-Paul FITOUSSI, Le débat interdit : monnaie, Europe, pauvreté, Paris, Éditions du Seuil, 2000 (1 $1^{\text {ère }}$ édition 1995), p. 311.

2.. Ibidem.

3.. Karl POLANYI, The Great Transformation, New York, Rinehart, 1944, traduction française La grande transformation. Aux origines politiques et sociales de notre temps, Paris, Éditions Gallimard, 1983, 419 p.

4.. Charles MORAZÉ, Les Bourgeois conquérants, Paris, Éditions Complexe, 1985 (1 ère édition 1957), 2 volumes, p. 408 et p. 2.

5.. Jean GADREY, Nouvelle économie, nouveau mythe ?, Paris, Éditions Flammarion, 2000, p. 12.

6.. Luc BOLTANSKI et Ève CHIAPELLO, Le nouvel esprit du capitalisme, NRF essais, Paris, Éditions Gallimard, 1999, 843 p.

7.. Max WEBER, Die protestantische Ethik und der Geist des Kapitalismus, dans Archiv für Sozialwissenschaft und Sozialpolitik, $\mathrm{n}^{\circ} 2,1905$, traduction française L'éthique protestante et l'esprit du capitalisme, Agora, Paris, Librairie Plon, 1964, 286 p.

8.. Luc BOLTANSKI et Ève CHIAPELLO, Le nouvel esprit du capitalisme, ouv. cité, p. 133.

9.. Ce texte, d'une richesse exceptionnelle, figure aux Archives nationales (arch. nat., 44 AQ 5) sous le titre Travail sur la fabrication des draps dans les archives Neuflize. J'en dois la connaissance à Gérard Gayot qui en prépare une publication complète. Voir Gérard GAYOT, Les draps de Sedan 1646-1870, Paris, Éditions de l'École des hautes études en sciences sociales/Éditions Terres ardennaises, 1998, notamment pp. 438-439.

10.. Alfred MOTTE-GRIMONPREZ, Lettres d'Alfred Motte-Grimonprez 1827-1887, s.l.s.d., 1952, 3 volumes. 
11.. Autre document que je dois à Gérard Gayot ; Archives de l'État à Liège, Archives d'entreprises, Fonds Peltzer, 100. Comme l'ensemble de ce fonds des entreprises de Verviers, il va être l'objet d'une analyse très prometteuse menée par Sylvie VaillantGabet.

12. Robert SALAIS et Michael STORPER, Les mondes de production. Enquête sur l'identité économique de la France, Paris, Éditions de l'École des hautes études en sciences sociales, 1993, $467 \mathrm{p}$.

13.. Travail en cours de Sylvie Vaillant-Gabet.

14.. A. DEWAVRIN, Un acheteur de laine en Afrique du Sud, s.e., 1999.

15.. Alfred D. CHANDLER Jr, The Visible Hand, Cambridge, Cambridge University Press, 1977, traduction française La Main visible des managers : une analyse historique, Paris, Éditions Économica, 1988, 635 p. L'auteur analyse et glorifie la substitution de managers aux propriétaires à la direction des entreprises.

16.. Alfred MOTTE-GRIMONPREZ, Lettres..., ouv. cité, volume 3, p. 604.

17. Gustave GOLDENBERG, ancien représentant à l'assemblée législative, De l'avenir de notre société, Paris, Librairie Nouvelle, 1856, 207 p.

18. Nicolas STOSKOPF, Les patrons du Second Empire, Alsace, Paris/Le Mans, Picard/

Cenomane, 1994, pp. 70-72.

19.. Maurice LÉVY-LEBOYER, « Le patronat français a-t-il été malthusien ? ", dans Le Mouvement Social, $\mathrm{n}^{\circ}$ 91, juillet-septembre 1974 ; Louis BERGERON, «Permanences et renouvellement du patronat », dans Yves LEQUIN [dir.], Histoire des Français XIX ${ }^{\mathrm{e}}-\mathrm{XX}^{\mathrm{e}}$ siècles, Paris, Librairie Armand Colin, 1983, 2 volumes, volume 2, pp. 153-292.

20.. Robert FITZGERALD, dans Youssef CASSIS, François CROUZET et Terry GOURVISH (ed.), Management and business in Britain and France; the age of the corporate economy, Oxford, Clarendon Press, 1995, 239 p.

21.. Max WEBER, L'éthique protestante..., ouv. cité, pp. 48-51.

22.. Adolphe THIERS, De la propriété, Paris, Paulin, Lheureux et Cie, 1848, p. 253.

23. Jean-Pierre HIRSCH, Les deux rêves du Commerce. Entreprise et institution dans la région lilloise (1780-1860), Paris, Éditions de l'École des hautes études en sciences sociales, 1991, chapitre 12.

24.. Arch. nat., F12, 2535.

25.. Gérard TASSIN, Le rôle du marché financier lillois dans le développement de l'économie du Nord-Pas de Calais de 1882 à 1914, Thèse d'histoire sous la direction d'Albert Broder, Université Paris 12, mai 2000, $1006 \mathrm{f}^{\circ}, \mathrm{f}^{\circ} 151$.

26.. Voir les travaux de Louis BERGERON depuis l'article : «A propos des biens nationaux : la signification économique du placement immobilier », dans Annales, Économies, sociétés, civilisations, $\mathrm{n}^{\circ} 2,1971$, pp. 415-419.

27. Guillaume Ternaux, créateur d'une vaste entreprise lainière, député en 1818, est mort à peu près ruiné en 1833. Jacques Laffitte, banquier, député en 1816, dirige le gouvernement en 1830-1831, doit liquider sa maison en 1836.

28. Cette partie du texte a déjà été publiée : voir Gérard GAYOT, « Le testament économique d'André de Neuflize, failli de haute lignée, 1836 », dans Entreprises et histoire, $\mathrm{n}^{\circ}$ 9, 1995, pp. 127-131.

29.. Alfred MOTTE-GRIMONPREZ, Lettres..., ouv. cité, volume 1, p.XLI.

30.. « Un nombre croissant d'entreprises quittent la Bourse », dans Le Monde, 21 février 2001.

31.. Entretien privé, réalisé en mars 1999.

32.. Exposées à des offres publiques d'achat sur le marché boursier. 
33.. Karl POLANYI, « Naissance du credo libéral », dans Karl POLANYI, La Grande Transformation..., ouv. cité, chapitre 12.

34.. Ibidem, pp. 196-197.

35.. Boisguillebert fut, au début du $18^{\mathrm{e}}$ siècle, un des tout premiers à énoncer l'idée d'un équilibre naturel des prix, obtenu par la libération des échanges et permettant d'atteindre un niveau maximum de production.

36.. Frédéric Bastiat, auteur en 1850 des Harmonies économiques et, la même année, d'une brochure où il explique par exemple que la concurrence que (les commerçants) se font entre eux les porte invinciblement à faire profiter les consommateurs de toutes les économies réalisées ; Frédéric BASTIAT, Harmonies économiques, Paris, Guillaumin, 1850, $463 \mathrm{p}$.

37.. Patrick VERLEY, L'échelle du monde, Essai sur l'industrialisation de l'Occident, Paris, Éditions Gallimard, 1997, 713 p. ; voir surtout la seconde partie ; citation p. 623.

38. Arch. nat., Centre des archives du monde du travail (Roubaix), 84AQ 13.

39.. Arch. nat., Centre des archives du monde du travail (Roubaix), 84AQ 35.

40.. François CARON, « Ententes et stratégies d'achat dans la France du XIX ${ }^{\mathrm{e}}$ siècle », dans Revue française de gestion, $\mathrm{n}^{\circ} 70$, septembre-octobre 1988. L'auteur ajoute à vrai dire qu'ils « n'ont cherché à en atténuer certains effets que pour préserver les intérêts des producteurs en place... ».

41.. Conseil supérieur de l'agriculture, du commerce et de l'industrie, Enquête, Traité de commerce avec l'Angleterre, Paris, Imprimerie impériale, 1860, tome 1, p. 196, et tome 3, p. 257.

42.. Cité par François CARON, « Ententes et stratégies d'achat ... », art. cité.

43.. Jean-Pierre HIRSCH, Les deux rêves..., ouv. cité, l'aborde pour la période 1780-1860.

44.. Alfred MOTTE-GRIMONPREZ, Lettres..., ouv. cité, volume 3, p. 655.

45.. Max WEBER, L'éthique protestante..., ouv. cité, p. 51.

46. Conclusion du Prospectus adressé aux journaux en 1817 : Claude-Henri de SAINT-SIMON, CEuvres, Paris, Éditions Anthropos, 1966, volume 2, p. 221.

\section{RÉSUMÉS}

Le capitalisme du XIX ${ }^{\mathrm{e}}$ siècle était-il plus " pur" que le nôtre et les discours tenus alors par les entrepreneurs et les théoriciens moins contradictoires? Analysant quelques échantillons de leurs écrits, à usage public ou à usage privé, les confrontant aux pratiques de leurs auteurs, on cherche à montrer la présence constante de contradictions ou d'alternatives qui semblent indispensables à la longévité du système. Autre époque! Mais sur la nature même de leur activité, la dimension familiale ou patrimoniale de leur entreprise, ou la place accordée à la concurrence, les discours des négociants et des industriels ne traduisaient pas moins de perplexité ou d'équivoques que celui des entrepreneurs d'aujourd'hui.

A look backwards on the older spirit of capitalism. Was $19^{\text {th }}$-century capitalism more "pure" than ours? And was the discourse then held by entrepreneurs and theoreticians less contradictory than it is now? While analysing some samples of their writings - be they with a 
public or with a private intent - and confronting these writings with the practises of their authors, this paper aims at pointing to the constant presence of contradictions or alternatives that seem to be indispensable to the long life of the system. These were other times! But on the nature of their activity, on the family or property dimension of their companies, or on competition, the discourse of $19^{\text {th }}$-century traders and manufacturers did not express less perplexity nor ambiguity than the discourse of today's businessmen.

\section{AUTEUR}

\section{JEAN-PIERRE HIRSCH}

Professeur à l'Université Lille 3 